\title{
Optical Coefficients and PL Spectra with Variation in Ga-content in GaSeTe Thin Films: Visible Spectrum
}

\author{
Neeru Chaudhary*, S.K. Tripathi, Navdeep Goyal ${ }^{*}$ \\ Department of Physics, Punjab University, 160014 Chandigarh, India
}

(Received 21 March 2016; published online 03 October 2016)

\begin{abstract}
The optical properties (transmission and emission) for $\mathrm{Ga}_{x} \mathrm{Se}_{85}{ }_{-x} \mathrm{Te}_{15}(x=0,2,6,10,15)$ semiconductor were studied using UV Visible spectra and PL Spectra of thermally evaporated thin films. Optical band gap is found to be decreasing with increased Ga-content in the compositions. Increase in refractive index, dielectric constants and absorption coefficient is observed in the wavelength region $550-850 \mathrm{~nm}$. The fall in the optical band gap is explained with chemical bond approach and electro negativity. The increase in refractive index $(n)$ is interpreted in association to the mean coordination number and cohesive energy. Direct band gap is found to be allowed in these thin films.
\end{abstract}

Keywords: Optical constants, Optical band gap, Semiconductors, Coordination number, Cohesive energy, Electronegativity.

\section{INTRODUCTION}

Today's fast changing requirements of engineering applications has paved the way to find newer materials. One of the fastest modes of communication i.e. optical fiber communication has led to the studies of materials with different optoelectronic properties and conductivities. Study of optical properties is useful in manufacturing devices such as solar cells, LEDs, various visual displays. Optical parameters of the material decide their usage in the fabrication of optoelectronic devices and optical mass storage media. One of the most interesting categories of materials is semiconductors, as their properties can be tuned according to their doping levels. In ternary semiconductors electrical conduction is predicted due to two processes, band conduction and hopping conduction. [1]. New form of semiconductors based on Selenium, Tellurium with third element such as $\mathrm{Ge}, \mathrm{Ga}, \mathrm{Bi}, \mathrm{Ag}, \mathrm{Sb}$ are used in numerous applications these days [2]. These materials absorb electromagnetic radiations which allows its use in optics and optoelectronics to fabricate optical elements, optical memories, microlences, wave-guides, holography, optical filters, bio and chemical sensors and in many more optical applications [3-12]. In the present work, optical transmission spectra and PL Spectra are taken to carry out calculations of optical coefficients of the system $\operatorname{Ga}_{x} \mathrm{Se}_{85}{ }_{-x} \mathrm{Te}_{15}(x=0, \quad 2, \quad 6, \quad 10,15)$. Swanepoel's method is applied to calculate optical constants [13].

\section{EXPERIMENTAL STUDIES}

\subsection{Preparation of Material}

Conventional technique of melting and then ice cooled quenching is used to synthesize $\mathrm{Ga}_{x} \mathrm{Se}_{85}{ }_{x} \mathrm{Te}_{15}(x=0,2,6,10,15)$ material samples. For this the pure elements \{Selenium pellets $3 \mathrm{~N}$; Tellurium $3 \mathrm{~N}$ and Gallium pellet $5 \mathrm{~N}\}$ were weighed in required atomic weight percentages, using Wensar ${ }^{\mathrm{TM}} \mathrm{MAB} 220$ model electronic weighing machine having resolution of $10^{-4} \mathrm{gm}$. For different compositions the weighed materials were melted inside the furnace at $800{ }^{\circ} \mathrm{C}$ for nearly 24 hours in vacuum sealed Quartz ampoules (length$12 \mathrm{~cm}$; internal dia. $8 \mathrm{~mm}$ ) followed by quenching in ice cooled water. The prepared ingots were crushed into fine powder. This bulk material was further used to prepare thermally evaporated thin films using a molybdenum boat on clean glass substrates under vacuum of $10^{-5}$ Torr. To achieve the metastable equilibrium the films were taken out of the deposition chamber after 24 hours.

\subsection{Characterization}

Bulk materials were characterized using powder Xray diffraction. The patterns of $\mathrm{Ga}_{x} \mathrm{Se}_{85}-x \mathrm{Te}_{15}(x=0,2$, $6,10,15)$ are shown in Fig. 1.

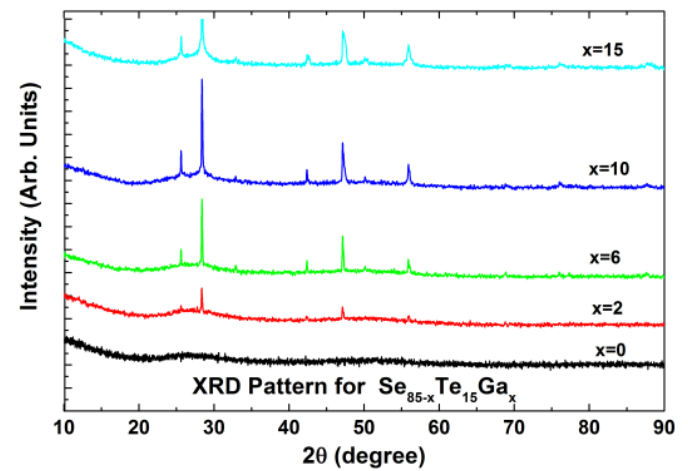

Fig. 1a - XRD of $\mathrm{Ga}_{x} \mathrm{Se}_{85-x} \mathrm{Te}_{15}(x=0,2,6,10,15)$ bulk form

X-Ray diffraction pattern shown confirms the amorphous nature of Se85 $\mathrm{Te}_{15}$ and polycrystalline nature of $\mathrm{Se}_{85}-x \mathrm{Te}_{15} \mathrm{Ga}_{x}(x=2,6,10$ and 15) alloys which has sharp and clearly resolved peaks. These measurements

\footnotetext{
*neeru369@pu.ac.in

†ngoyal@pu.ac.in
} 

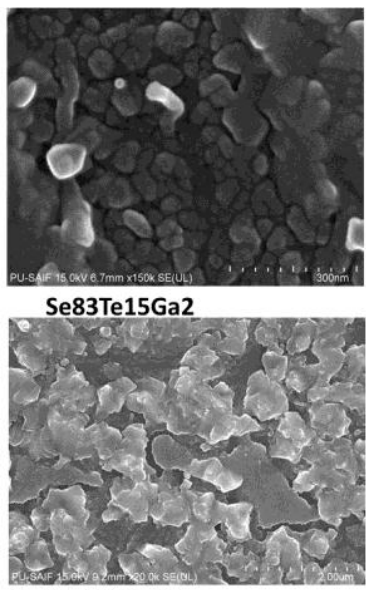

Se75Te15Ga10

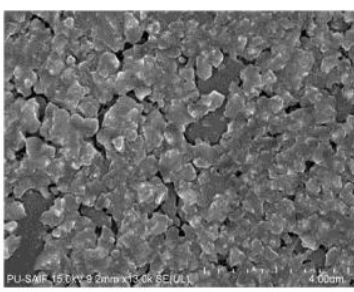

Se79Te15Ga6

Se70Te15Ga15

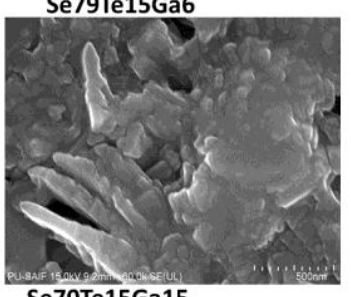

Fig. $1 \mathbf{b}-$ FESEM of $\mathrm{Se}_{85-x} \mathrm{Te}_{15} \mathrm{Ga}_{x}$ thin films

were done using $\mathrm{Cu} \mathrm{K}_{\mathrm{a}}$ line radiation of wavelength $1.54 \AA$. The average size of the crystallite was determined using Debye-Scherrer's formula: $D=0.9 \lambda / \beta \cos \theta$ where $\beta$ is the full width at half maximum (FWHM) of the peak, $\lambda$ is X-ray wavelength, $\theta$ is the Bragg angle. The average crystallite size of prepared compounds are less than $100 \mathrm{~nm} \mathrm{Gax} \mathrm{Te}_{15} \mathrm{Se}_{85}-x$ FESEM of thin films clearly shows the small crystals.

\subsection{UV/VIS \& PL Spectra}

A Double UV/VIS Perkin Elmer UV WinLab is used to take UV/VIS Spectra of $\mathrm{Ga}_{x} \mathrm{Se}_{85}-x \mathrm{Te}_{15}$ thin films in the wavelength region 500-2500 $\mathrm{nm}$. The PL mapping of thin films was done using a WITech alpha 300R+ confocal PL microscope system, where $375 \mathrm{~nm}$ diode laser is used as a source of excitation.

\section{RESULTS AND DISCUSSION}

\subsection{Optical Constants}

The transmission spectra of the thin films are given in Fig. 2. Red shift is observed in the absorption edge of the deposited films as there is increase in the Ga content in the compositions. This shift signifies the compositional dependence of the optical band gap of the material. Swanepoel method is used to derive the optical constants [13]. The optical constants are derived from the fringe patterns in the transmittance spectrum. For absorption coefficient $(\alpha=0)$ i.e. in the transparent region the refractive index $(n)$ is:

$$
n=\left[M+\left(M^{2}-s^{2}\right)^{1 / 2}\right]^{1 / 2}
$$

$$
\text { Where } \quad M=\left[\left(\frac{2 s}{T_{m}}\right)\right]-\frac{\left[s^{2}+1\right]}{2}, \quad T_{m}-\text { envelope }
$$

function of the transmittance minima $s$ - refractive index of the substrate.

In the region of weak and medium absorption, where $(\alpha \neq 0)$, absorption coefficients $(\alpha)$ and the refractive index $(n)$ is:

$$
n=\left[M+\left(M^{2}-s^{2}\right)^{1 / 2}\right]^{1 / 2}
$$

Where $M=\left[\left(\frac{2 s\left(T_{M}-T_{m}\right)}{T_{M} T_{m}}\right)\right]+\frac{\left[s^{2}+1\right]}{2}, T_{M}$ - enve-

lope function of the transmittance maxima.

In the strong absorption region the interference fringes disappear. There the values of $n$ can be estimated by extrapolating the calculated values in other parts of the spectrum. Using eqn. (1) and (2) the refractive index $(n)$ of the films can be calculated from the fringes shown in Transmission spectrum (Fig. 2).The extinction coefficient $(k)$ can be determined from the relation,

$$
k=\alpha \lambda /(4 \pi)
$$

The change in refractive index $(n)$ and extinction coefficient $(k)$ with variation in wavelength for $\mathrm{Ga}$ $\operatorname{Se}_{85}{ }_{x} \mathrm{Te}_{15}(x=0,2,6,10,15)$ are shown in Fig 3 and Fig 4 respectively. The speed of light in a material is dependent on electrical and magnetic properties of the material. Thus the deviation in real and imaginary part of the dielectric constant of the material Figs. 5 and 6 are in unison with the variation in Refractive index $(n)$. These values at a particular wavelength are given in Table 1 which depicts their increase with increased value of Ga-content in the compositions. Increase in the refractive index with increase in Ga content may be related with the increase of the mean coordination number of semiconductor materials [14-15]. FESEM shown in Fig $1 \mathrm{~b}$ also supports that as the Ga content is increased, crosslinking increases which is due to increase in the coordination number. The mean coordination number $\left(N_{c}\right)$ values for the studied ternary semiconductor alloy $\mathrm{Se}_{x} \mathrm{Te}_{y} \mathrm{Ga}_{z}(x+y+z=1)$, are calculated using the formula[16]:

$$
\mathrm{N}_{c}=x \mathrm{CN}(\mathrm{Se})+y \mathrm{CN}(\mathrm{Te})+z \mathrm{CN}(\mathrm{Bi})
$$

Where $\mathrm{CN}$ is the coordination number of the pure element given in Table 2 and $x, y$ and $z$ are the atomic concentrations of Se, Te and Ga, respectively.

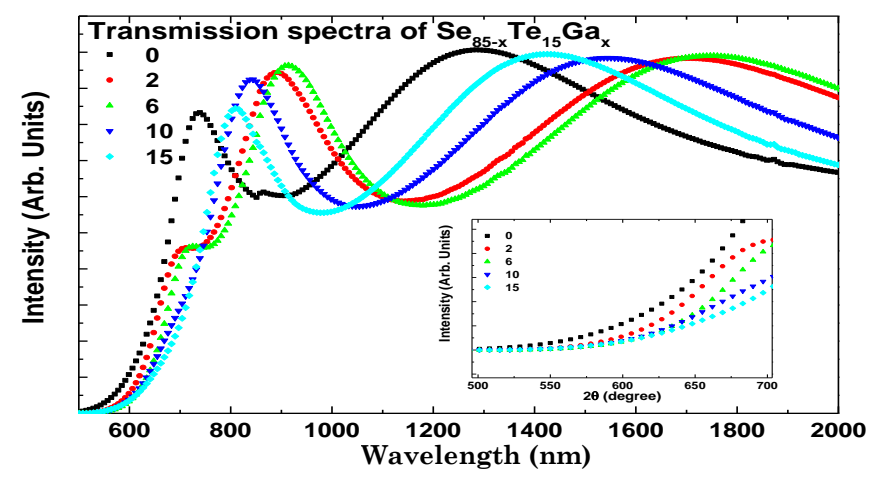

Fig. 1 - Transmission spectra of $\mathrm{Ga}_{x} \mathrm{Se}_{85-x} \mathrm{Te}_{15}(x=0,2,6,10$, and 15) 
Table 1 - Calculated values of absorption coefficient, refractive index, extinction coefficient, real and imaginary dielectric constant, mean coordination number and electronegativity

\begin{tabular}{|l|l|l|l|l|l|l|l|}
\hline $\begin{array}{l}X \\
\%\end{array}$ & $\begin{array}{l}\text { absorption } \\
\text { Coefficient }(\alpha) \\
690 \mathrm{~nm} \text {. in }\left(\mathrm{m}^{-1}\right)\end{array}$ & $\begin{array}{l}\text { Refrac- } \\
\text { tive } \\
\text { index }(n)\end{array}$ & $\begin{array}{l}\text { Extinction } \\
\text { coeffi- } \\
\text { cient }(k)\end{array}$ & $\begin{array}{l}\text { Real } \\
\text { dielectric } \\
\text { constant }\left(\epsilon^{\prime}\right)\end{array}$ & $\begin{array}{l}\text { Imaginary } \\
\text { Dielectric } \\
\text { Constant }\left(\epsilon^{\prime \prime}\right)\end{array}$ & $\begin{array}{l}\text { Mean Cor- } \\
\text { dination } \\
\text { number }\end{array}$ & $\begin{array}{l}\text { Electroneg- } \\
\text { ativity }\end{array}$ \\
\hline 0 & 4936 & 2.4 & 0.0271 & 5.70 & 0.13 & 2.00 & 2.48 \\
\hline 2 & 7590 & 3.0 & 0.0417 & 9.24 & 0.25 & 2.02 & 2.46 \\
\hline 6 & 8849 & 3.4 & 0.0486 & 11.44 & 0.33 & 2.06 & 2.43 \\
\hline 10 & 11414 & 4.2 & 0.0627 & 17.59 & 0.53 & 2.10 & 2.39 \\
\hline 15 & 12879 & 4.8 & 0.0708 & 22.59 & 0.67 & 2.15 & 2.35 \\
\hline
\end{tabular}

Table 2 - Values of atomic radius, coordination number, electronegativity and homo-nuclear bond energy of pure elements

\begin{tabular}{|c|c|c|c|c|}
\hline Element & Atomic Radius (pm) & $\begin{array}{l}\text { Coordination } \\
\text { number }\end{array}$ & Electronegativity & $\begin{array}{l}\text { Homo-nuclear bond } \\
\text { energy (Kcal/mol) }\end{array}$ \\
\hline $\mathrm{Se}$ & 117 & 2 & 2.55 & 44.0 \\
\hline $\mathrm{Te}$ & 137 & 2 & 2.10 & 33.0 \\
\hline $\mathrm{Ga}$ & 153 & 3 & 1.81 & 26.9 \\
\hline
\end{tabular}

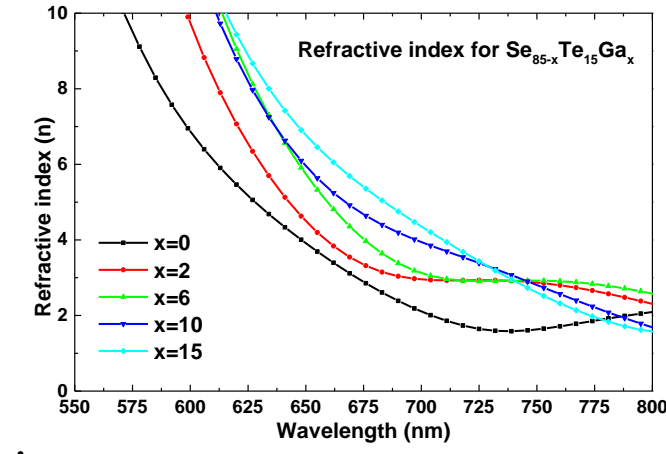

Fig. 2 - Wavelength v/s Refractive index (n) w.r.t. Ga atomic weight percentage variation.

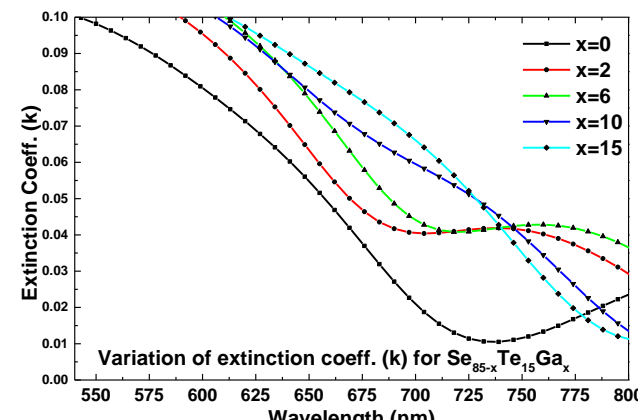

Fig. 3 - Wavelength v/s extinction coefficient $(k)$ w.r.t. Ga atomic weight percentage variation.

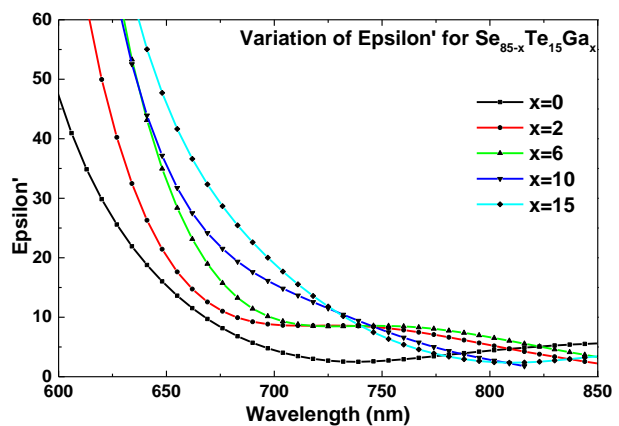

Fig. 4 - Wavelength v/s $\epsilon^{\prime}$ w.r.t. Ga atomic weight percentage variation

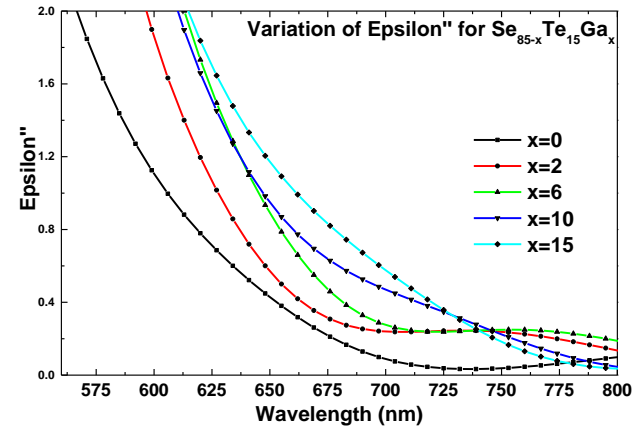

Fig. 5 - Wavelength v/s $\epsilon^{\prime \prime}$ w.r.t. Ga atomic weight percentage variation.

\subsection{Absorption Coefficient and Optical Band Gap}

The absorption coefficient $(\alpha)$ of $\mathrm{Ga}_{x} \mathrm{Se}_{85}{ }_{-x} \mathrm{Te}_{15}(x=0,2$, $6,10,15)$ thin films can be calculated using the relation

$$
\alpha=4 \pi k / \lambda
$$

Fig. 7 depicts dependence of the absorption coefficient, on photon energy $(h v)$; it is apparent that the value of the absorption coefficient increases for Ga rich compositions. The optical band gap $\left(E_{g}\right)$ of the films is obtained by fitting the absorption data in the Tauc's relation. Fig. 8 shows plot between $(\alpha h v)^{2}$ vs. photon energy $(h v)$. For the direct transition the values of $E_{g}$ were taken as the intercept of $(\alpha h v)^{2}$ vs. $(h v)$ at $(\alpha h v)^{2}=0$. It is assumed that due to increase in crystallinity, the defect states might have ordered their positions in the band structure resulting in direct band gap. The values of $E_{g}$ for different compositions are given in Table 3.Thevalue of Eg decreases with increasing Ga content.

This decrease in the value of $E_{g}$ can be explained in the light of cohesive energy and chemical bond approach and electronegativity. In a multi-component system the position of conduction and valence band edges as well as the energy gap depends on the number of various possible bonds in the system and the average bond energy. The heteronuclear bond energies $D(A-B)$ are calculated with the relation [17]: 

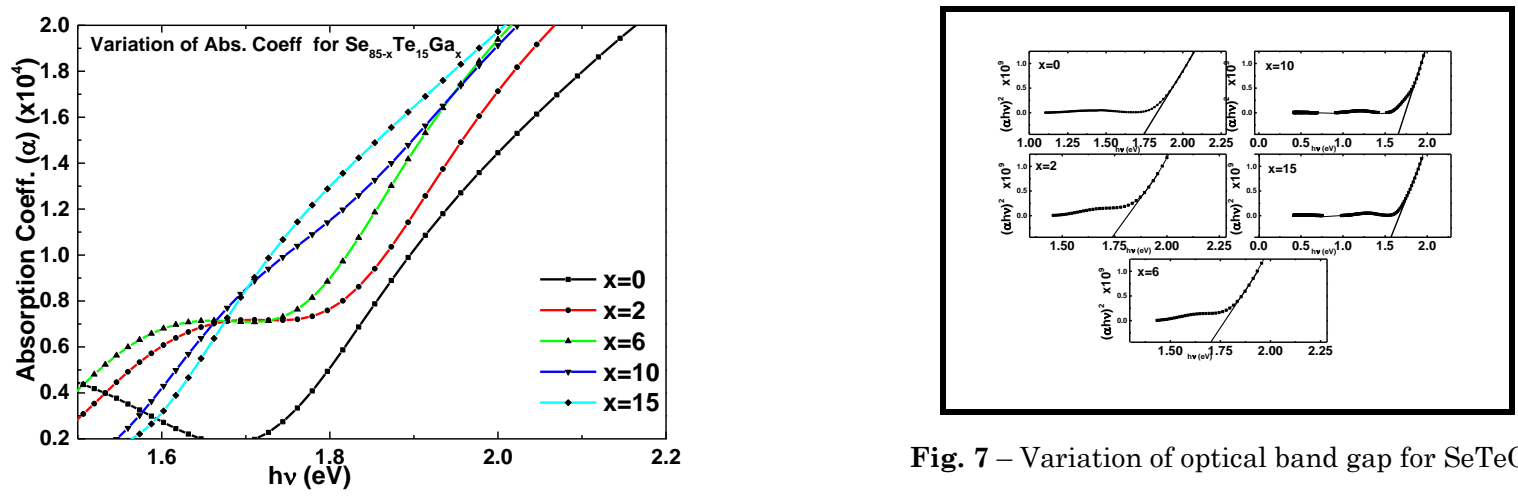

Fig. $6-h v(E)$ v/s absorption coefficient w.r.t. Ga atomic

Fig. 7 - Variation of optical band gap for SeTeGa weight percentage variation

Table 3 - The bond probabilities in $\mathrm{Ga}_{x} \mathrm{Se}_{85}{ }_{-x} \mathrm{Te}_{15}(x=0,2,6,10,15)$ and cohesive energy

\begin{tabular}{|l|l|l|l|l|c|}
\hline \multirow{2}{*}{ Composition } & \multicolumn{3}{|c|}{ Bonds Formed } & \multirow{2}{*}{$\begin{array}{c}\text { Cohesive energy } \\
\text { (kcal/mol) }\end{array}$} & $\begin{array}{c}\text { Optical band } \\
\text { gap }\end{array}$ \\
\cline { 2 - 4 } & Se-Ga & Se-Te & Se-Se & & $E_{g}(\mathrm{eV})$ \\
\hline Se$_{85} \mathrm{Te}_{15}$ & - & 0.1765 & 0.8235 & 44.03 & 1.75 \\
\hline $\mathrm{Se}_{83} \mathrm{Te}_{15} \mathrm{Ga}_{2}$ & 0.0361 & 0.1807 & 0.7831 & 44.29 & 1.73 \\
\hline $\mathrm{Se}_{79} \mathrm{Te}_{15} \mathrm{Ga}_{6}$ & 0.1139 & 0.1898 & 0.6962 & 44.86 & 1.70 \\
\hline $\mathrm{Se}_{75} \mathrm{Te}_{15} \mathrm{Ga}_{10}$ & 0.2000 & 0.2000 & 0.6000 & 45.49 & 1.65 \\
\hline $\mathrm{Se}_{70} \mathrm{Te}_{15} \mathrm{Ga}_{15}$ & 0.3214 & 0.2142 & 0.4642 & 46.37 & 1.57 \\
\hline
\end{tabular}

$$
\begin{aligned}
& D(A-B)=[D(A-A) \times D(B-B)]^{1 / 2}+ \\
&+30(x A-x B)^{2}
\end{aligned}
$$

$D(A-A)$ and $D(B-B)$ are the homonuclear bond energies, $x A$ and $x B$ are values of electronegativity for atoms involved [18] and are listed in Table 2. Chemical bond approach suggests that bonds are formed in the order of decreasing bond energies till the accessible valences of atoms is satisfied [18]. In these compositions, the Se-Ga bonds having highest heteronuclear bond energy (51.278 $\mathrm{kcal} \mathrm{mol}^{-1}$ ) are predicted to crop up first followed by the Se-Te bonds with $44.18 \mathrm{kcal}$ $\mathrm{mol}^{-1}$ bond energy to saturate all available valences of Se. After the formation of these bonds, there are still unsatisfied Se valences, to be satisfied by the formation homonuclear Se-Se bonds. The cohesive energy is calculated by chemical bond approach [19]

$$
C E=\sum C_{i} D_{i}
$$

$C_{i}$ - Expected number of chemical bonds;

$D_{i}$ - Corresponding bond energy.

Calculated values of the $C E$ and the probability of chemical bonds formed are given in Table 3. The outcome reflects that the cohesive energy of the studied system increases with increased $\mathrm{Ga}$ content in the compositions. Even with the increase in cohesive energy, the decrease in optical band gap is observed. This may be attributed to replacement of Selenium with Gallium i.e. addition of less electronegative elements to the compositions may increase the energy of lone pair states, thus broadening of valence band inside the forbidden gap leading to decrease in the optical band gap. Also, electronegativity of the prepared compositions (Table 2) is decreasing which is calculated by Sanderson's principle [20].As per this principle, electronegativity of an alloy is calculated as the geometric mean of electronegativity of its component elements. It is ob- servable from the Table 2 that the value of electronegativity decreases with increase in Ga content. Electronegativity is measure of the power to exert a pull on electron towards itself in a molecule. The relationship of the electron affinity with the optical band gap was studied by various researchers [21-22]. Mulliken [23], states that the average of the ionization potential and the electron affinity is electronegativity. It is not possible to allocate an electron affinity value for a semiconducting alloy. Thus, for this studied system it will be suitable to link the electronegativity with optical band gap. In this system, it is experiential that with decreasing electronegativity of a sample the optical band gap decreases. Also, there may be shift in the Fermi level due to addition of conductive element.

\subsection{PL Spectra}

The luminescence studies were performed on thin films by exciting at $375 \mathrm{~nm}$. The colours of the visible spectrum can be broken up into the approximate wavelength values The PL Spectra shown in Fig. 9 shows emission of visible wavelength in Red region (Red 610 to approximately $750 \mathrm{~nm}$ ) [24]. $\mathrm{Se}_{85} \mathrm{Te}_{15}$ has maximum emission in the given wavelength. The peaks observed are at $620 \mathrm{~nm}$ and $658 \mathrm{~nm}$. It is possible that the recombination of some of the free excitons becomes radiative by going from amorphous to crystalline phase [25]. As Gallium is inserted in the system it shows quenching of the emission. But as the Gallium content increases it again shows emission in the same wavelength region as crystallinity increases. It depicts Gallium is not changing the wavelength but varies the intensity of particular wavelength emission. This clearly signifies the increase in defect states. Gallium has led to better cross-linked system but increased defect states. Band gap is decreasing with increased Ga- 
content but the same result is not observed in the emission spectrum.

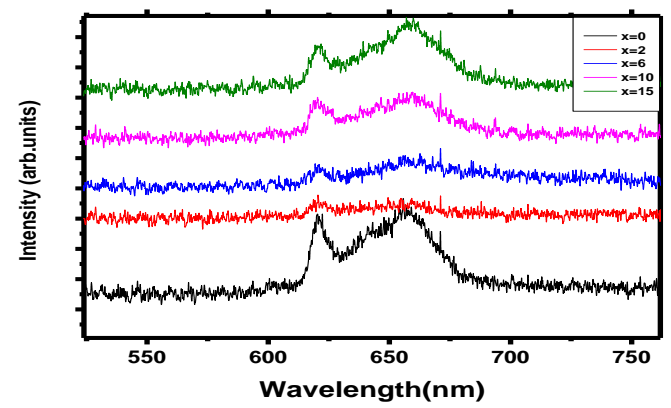

Fig. 9 - PL emission spectrum (500-780 $\mathrm{nm}$ visible region) of $\mathrm{Ga}_{x} \mathrm{Se}_{85}-x \mathrm{Te}_{15}(x=0,2,6,10,15)$ thin films at an excitation of $375 \mathrm{~nm}$

\section{CONCLUSION}

Optical Coefficients were obtained for $\mathrm{Ga}_{x} \mathrm{Se}_{85}{ }_{-x} \mathrm{Te}_{15}$ using the transmittance. The method recommended by

\section{REFERENCES}

1. N.F. Mott, E.A. Davis, Electron Processes in NonCrystalline Materials, Clarendon Press, Oxford (1979).

2. Abhay Kumar Singh, Rev. Adv. Sci. Eng. 1, 292 (2012).

3. S.A. Khan, M. Zulfequar, M. Hussain, Vacuum 72, 291 (2003).

4. T.Z. Babeva, D. Dimitrov, S. Kitova, I. Konstantinov, Vacuum 58, 96 (2000)

5. I.V. Mikla, I.P. Mikhalko, V.V. Mikla, Mater. Sci. Eng. B 83, 74 (2001)

6. M. Frumar, T. Wagner, Curr. Opin. Sol. State Mater. Sci. 7, 117 (2003)

7. R.V. Woudenberg, Jpn. J. Appl. Phys. 37, 2159 (1998).

8. K. Tanaka, J. Non-Cryst. Solids 1179, 164 (1993).

9. T. Kawaguchi, S. Maruno, S.R. Elliot, J. Appl. Phys. 79, 9096 (1996).

10. M. Ohto, phys. status solids a 159, 461 (1997).

11. T. Wagner, M. Frumar, V. Suskova, J. Non-Cryst. Solids 128, 197 (1991).

12. A.K. Kolobov, J. Tominaga, J. Optoelectron. Adv. Mater. 4 (3), 679 (2002)
Swanepoel for thin films having reasonable number of interference fringes is applied. Energy gap decreases with increasing Ga-content. The decrease in Energy gap is explained in reference to chemical bond approach and electronegativity. The rise in refractive index, dielectric constants and absorption coefficient in the wavelength range $550-850 \mathrm{~nm}$ is explained on the basis of the increase in the density of the material with Ga content and mean coordination number. The direct band gap is the result of increase in crystallinity with increase in Ga content in the compositions. The PL emission is observed in visible red region. The core and cladding materials are selected on the basis of their refractive indices. In the studied system, at a particular wavelength the material doped with different $\mathrm{Ga}$ atomic weight percentage shows different refractive index. Moreover, it shows direct band gap hence, points towards its application in fabricating optical devices. Moreover absorption coefficient increases with Ga content which makes it suitable for optical memory storage devices.

13. R. Swanepoel, J. Phps. E: Sci. Instrum. 16, 1983 (1983)

14. E.R. Shaaban, Yasser A.M. Ismaila, H. Shokry Hassan, J. Non-Crystalline Solids 376, 61 (2013).

15. J.C. Phillips, J. Non-Cryst. Solids 34, 153 (1979) (J.C. Phillips, Solid State Commun. 53, 699 (1985)).

16. T.S. Moss, Optical Properties of Semiconductors (Buttenworths: London: 1959).

17. L. Pauling, Nature of the Chemical Bonds (Cornell University Press: Ithaca: New York: 1960)

18. K. Sedeek, M. Fadel, Thin Solid Films 229, 223 (1993).

19. B. Jozef, O. Stanford, S. Mahadevan, A. Gridhar, A.K. Singh, J. Non-Cryst. Solids 74, 75 (1985).

20. R.T. Sanderson, Inorganic Chemistry, Third Edition (London, New York: 1991).

21. F.P. Koffeyberg, J. Phys. Chem. Solids 53, 1285 (1992).

22. A.A. Al-Ghamdi, Vacuum 80, 400 (2006).

23. R.S. Mulliken, J. Chem. Phys. 2, 782 (1984).

24. J. Reichman, Handbook of Optical Filters for Fluorescence Microscopy (Chroma Technology: Brattleboro: 2010)

25. Salah, Nanoscale Res. Lett. 7, 509 (2012). 